\title{
Neutrino Masses and See-Saw Mechanism
}

\author{
Ahmad Tarhini, Mohamad Mrad \\ Faculty of Sciences V, Physics Department, Lebanese University, Nabatieh, Lebanon \\ Email: tarhini.ahmad@gmail.com, mohamadmrad84@gmail.com
}

How to cite this paper: Tarhini, A. and Mrad, M. (2020) Neutrino Masses and See-Saw Mechanism. Journal of High Energy Physics, Gravitation and Cosmology, 6, 274-281.

https://doi.org/10.4236/jhepgc.2020.62021

Received: March 29, 2020

Accepted: April 23, 2020

Published: April 26, 2020

Copyright (c) 2020 by author(s) and Scientific Research Publishing Inc. This work is licensed under the Creative Commons Attribution International License (CC BY 4.0).

http://creativecommons.org/licenses/by/4.0/

\section{(c) (i) Open Access}

\begin{abstract}
A neutrino is a subatomic particle that is very similar to an electron, but has no electrical charge and a very small mass. Neutrinos are one of the most abundant particles in the universe. Because they have very little interaction with matter, however, they are incredibly difficult to detect. We present a study of the physics of neutrinos using the Dirac lagrangian. Based on Lorentz invariance we introduce the notion of Majorana spinor. Then we derive the mass terms for both Dirac and Majorana neutrinos. We further discuss the general framework of the See-Saw mechanism considering a simplification of the problem.
\end{abstract}

\section{Keywords}

Neutrinos, Lagrangian and Dirac Equation, Majorana Spinors, Massive Neutrinos, See-Saw Mechanism

\section{Introduction}

The neutrino is an elementary particle of the standard model of particle physics. It's a fermion of spin 1/2. The existence of the neutrino was postulated by Pauli in 1930, in order to explain the non-conservation of energy in beta decay, but its experimental discovery did not occur until 1956, when Reins and Cowan revealed the interactions of neutrinos from the Savannah River nuclear reactor [1] [2].

The study of the properties of neutrinos still presents many unsolved problems, and in particular the fundamental question on the mass of the neutrinos. In this context, the study of neutrino oscillations plays a privileged role, because their observation implies a non-zero mass. Oscillations are the key to solving different experimental observations, such as the problem of solar neutrinos. In 1962, the muonic neutrino $v_{\mu}$ is discovered at Brookhaven and the tau neutrino $v_{\tau}$ in 2000 at DONUT experiment. In 1990, the LEP at CERN shows that 
there are three families of light neutrinos (mass $\leq 45 \mathrm{GeV}$ ): $v_{e}, v_{\mu}$ and $v_{\tau}$. Some experiments, notably that of Super-Kamiokande in 1998 (Nobel Prize in 2002 on this occasion), have shown that neutrinos can, through a phenomenon called neutrino oscillation, be continuously transformed from one form of flavor (electronic, muonic or tau) into another. The discovery of this phenomenon made it possible to provide a solution to the problem of solar neutrinos [3] [4].

With this in mind, in the current context, starting from the above introduction, this paper is organized as follows: In Section 2 we introduce the Dirac lagrangian and the corresponding equations of motion. Then in Section 3 we explain the Majorana spinor in addition to the difference with Dirac spinor. In Section 4, we shall explore and discuss the neutrino mass term with a detailed calculation. In Section 5, we will show and derive the equations of the See-Saw mechanism, and Section 6 is devoted to our conclusions.

\section{The Dirac Equation}

Dirac's equation is a relativistic quantum mechanics equation that describes half-integer spin particles [5]. The Dirac lagrangian is given by:

$$
L=\bar{\psi}(x)\left(i \gamma^{\mu} \partial_{\mu}-m \cdot \mathbb{I}\right) \psi(x),
$$

where $\bar{\psi}(x)=\psi^{\dagger}(x) \gamma^{0}, \gamma^{\mu}$ is the Dirac matrix which is a Lorentz vector $\gamma^{\mu}=\left(\gamma^{0}, \gamma^{i}\right), \quad \gamma^{0}=\left(\begin{array}{cc}\mathbb{I}_{2 \times 2} & 0_{2 \times 2} \\ 0_{2 \times 2} & -\mathbb{I}_{2 \times 2}\end{array}\right)$ and $\gamma^{i}=\left(\begin{array}{cc}0_{2 \times 2} & \sigma_{i} \\ -\sigma_{i} & 0_{2 \times 2}\end{array}\right)$, where $\sigma_{i}$ are Pauli matrices.

From now on, the 4-dimensional unit matrix and the vector $(x)$ of Minkowski space-time will not be marked. One can divide this lagrangian into a kinetic term and another massive term:

$$
L=L_{\text {kinetic }}+L_{\text {massive }}=i \bar{\psi} \gamma^{\mu} \partial_{\mu} \psi-m \bar{\psi} \psi .
$$

From this Lagrangian, valid for a global symmetry $U(1)$, we can use the Euler-Lagrange equation to obtain the Dirac equation and its conjugate.

For $\psi$ :

$$
\partial_{\mu}\left(\frac{\partial L}{\partial_{\mu} \psi}\right)-\frac{\partial L}{\partial \psi}=0,
$$

where $\frac{\partial L}{\partial \psi}=-m \bar{\psi}$ and $\frac{\partial L}{\partial_{\mu} \psi}=\bar{\psi} i \gamma^{\mu}$; we then obtain the conjugate Dirac equation:

$$
\partial_{\mu}\left(\bar{\psi} i \gamma^{\mu}\right)+m \bar{\psi}=0
$$

For $\bar{\psi}$ :

$$
\partial_{\mu}\left(\frac{\partial L}{\partial_{\mu} \bar{\psi}}\right)-\frac{\partial L}{\partial \bar{\psi}}=0
$$

we obtain the Dirac equation: 


$$
\left(i \partial_{\mu} \gamma^{\mu}-m\right) \psi=0
$$

\section{Majorana Spinors}

It is a fermionic type particle which is its own antiparticle. The neutrino could be either a Majorana or Dirac particle. This implies that the spinor $\psi$ is related to its conjugate $\psi^{*}$. In fact, the equation $\psi=\psi^{*}$ is not Lorentz invariant, then the solution is to use the charge conjugate spinor [3] [4] [6]. This gives a definition of Majorana Fermions compatible with the Lorentz invariance:

$$
\psi_{M}=\mathrm{e}^{i \delta} \psi_{M}^{c}, \quad \forall \delta \in \mathfrak{R},
$$

then, using the Majorana representation [5] and the charge conjugate matrix ${ }^{1}, C$, we obtain:

$$
\psi_{M}^{c}=C_{M} \gamma_{M}^{0} \psi_{M}^{*}=\mathrm{e}^{i \theta}\left(\gamma_{M}^{0}\right)^{2} \psi_{M}^{*}=\mathrm{e}^{i \theta} \psi_{M}^{*},
$$

and $\psi_{M}^{c}=\psi_{M} \mathrm{e}^{-i \theta}$ then for $\delta=-\theta$, we obtain $\psi_{M}=\psi_{M}^{*}$. Hence the four components of the Majorana spinor are real, and it has half the degrees of freedom of a Dirac spinor.

\section{The Massive Neutrino}

\subsection{Mass Terms}

Charged leptons and quarks, i.e. all fermions of the standard model of particle physics apart from the neutrinos, are Dirac particles. It is tempting to think that neutrinos are too. A 4-component Dirac spinor represents the particle and the antiparticle, each in the states of left and right chirality respectively. When a neutrino is considered massless, the standard model doesn't contain the right chirality field $v_{R}$, but only the left chirality field $v_{L}$. So, in order to introduce the mass of the neutrino as the mass of the introduced quarks, we add $v_{R}$ to the model [6] [7].

Starting from the massive term of Dirac lagrangian, $L_{\text {massive }}=-m \bar{\psi} \psi$, and knowing that $\psi=P_{L} \psi+P_{R} \psi$, then $\psi_{L}=P_{L} \psi \Rightarrow \overline{\psi_{L}}=\overline{P_{L} \psi}=\left(P_{L} \psi\right)^{+} \gamma^{0}=\psi^{+} P_{L}^{+} \gamma^{0}$ since $\bar{\psi}=\psi^{+} \gamma^{0}$.

Using the proprieties of the projection operator and the $\gamma$ matrices, we obtain $P_{L}^{+}=P_{L}$ and

$$
P_{L}^{+} \gamma^{0}=\frac{1}{2}\left(1+\gamma^{5}\right) \gamma^{0}=\frac{1}{2} \gamma^{0}\left(1-\gamma^{5}\right)=\gamma^{0} P_{R} \Rightarrow \overline{\psi_{L}}=\psi^{+} \gamma^{0} P_{R}=\bar{\psi} P_{R} .
$$

Then $L_{m_{D}}=-m \bar{\psi} \psi=-m\left(\overline{\psi_{L}+\psi_{R}}\right)\left(\psi_{L}+\psi_{R}\right)=-m\left(\overline{P_{L} \psi+P_{R} \psi}\right)\left(P_{L} \psi+P_{R} \psi\right)$, so

$$
L_{m_{D}}=-m\left(\bar{\psi} P_{R}+\bar{\psi} P_{L}\right)\left(P_{L} \psi+P_{R} \psi\right)=-m\left(\overline{\psi_{L}} \psi_{R}+\overline{\psi_{R}} \psi_{L}\right)=-m \overline{\psi_{L}} \psi_{R}+\text { h.c } .
$$

That's give the Dirac term where $m_{D}$ is a constant. Consequently, one can construct the Dirac mass term:

${ }^{1}$ Charge conjugate matrix: This matrix is used to define the charge conjugate spinor $\psi^{c}(x)=C \gamma^{0} \psi^{*}(x) . C$ is defined within a phase factor, $C=\mathrm{e}^{i \theta} \gamma^{2} \gamma^{0}, \forall \theta \in \mathfrak{R}$. 


$$
L_{m_{D}}=-m_{D} \bar{v}_{L} v_{R}+\text { h.c. }
$$

$L_{m_{D}}$ can be written in matrix form:

$$
L_{m_{D}}=-m_{D}\left(\bar{v}_{L} v_{R}+\bar{v}_{R} v_{L}\right)=-\frac{1}{2}\left(\bar{v}_{L} \bar{v}_{R}\right)\left(\begin{array}{cc}
0 & m_{D} \\
m_{D} & 0
\end{array}\right)\left(\begin{array}{c}
v_{L} \\
v_{R}
\end{array}\right)+\text { h.c. }
$$

where $m_{D}$ is a complex $(3 \times 3)$ matrix. This term is invariant under the global transformation $U(1)$ :

$$
v_{\alpha} \rightarrow \mathrm{e}^{i \phi} v_{\alpha}, \quad \alpha \rightarrow \mathrm{e}^{i \phi} \alpha \quad(\alpha=e, \mu, \tau),
$$

where the phase $\phi$ is the same for all neutrinos and all charged leptons. This implies the conservation of the total leptonic number $L$. Thus, $L$ is the quantum number which distinguishes a neutrino from an antineutrino.

A massive fermion must have both left and right components: $\psi=\psi_{L}+\psi_{R}$. However there are two possibilities: in a case, the right component is completely independent of the left component, we then have a Dirac field. In a second case, the right field is the conjugate of the left field:

$$
\psi_{R}=\left(\psi_{L}\right)^{c} \text {, }
$$

where $\hat{C}$ is charge conjugate operator (particle-antiparticle).

Using the proprieties of commutation of Dirac $\gamma$ matrices, we show that the operator $\hat{C}$ applied to a chiral field toggles its chirality:

$$
\left(\psi_{L}\right)^{c}=\left(\psi^{c}\right)_{R}, \quad\left(\psi_{R}\right)^{c}=\left(\psi^{c}\right)_{L},
$$

then

$$
\psi_{R}=\left(\psi_{L}\right)^{c}=\left(\psi^{c}\right)_{R}
$$

Once $v_{R}$ is added to the model description, we introduce the Majorana mass term:

$$
L_{M}=-m_{R} \bar{v}_{R}^{c} v_{R}+\text { h.c., }
$$

where $m_{R}$ is another constant. This term mix $v$ and $\bar{v}$ and doesn't conserve the leptonic number $L$.

Note that there is a very important difference between the Dirac and Majorana terms. While the first preserves the electric charges, the baryonic and leptonic numbers; the second (i.e. Majorana term) violates the conservation of all additive quantum numbers of two units.

Since the electric charge is exactly conserved, this means that no charged particle can be of Majorana type. So among all fermions, only neutrinos can be described by Majorana fields, and in this case the leptonic number will be violated.

\subsection{Partners of Neutrino Masses}

The Dirac term, already introduced, conserves the fermionic numbers

$$
\psi \rightarrow \mathrm{e}^{i \alpha} \psi, \quad \bar{\psi} \rightarrow \mathrm{e}^{-i \alpha} \bar{\psi},
$$

and gives equal masses for particles and antiparticles $m_{\bar{\psi}}=m_{\psi}=m$. For par- 
ticles obeying $U(1)$ symmetry, such as the electromagnetic charge, it's clear that $L_{m_{D}}$ is the only possible mass term. Then, to conserve $U(1)$ symmetry, we always need a particle-antiparticle interaction [8].

Since neutrinos do not have electromagnetic charges, it is possible to introduce another mass term that contains two fields of neutrinos (or antineutrinos). The general mass term contains then the Dirac mass term in addition to the Majorana term which has a left and right contributions [9]. It can be written in the following form:

$$
\begin{aligned}
L_{\text {masse }}^{v}= & -\left[\bar{v}_{R} m_{D} v_{L}+\bar{v}_{L} m_{D}^{\dagger} v_{R}\right]-\frac{1}{2}\left[\bar{v}_{R} \tilde{C} m_{S} \bar{v}_{R}^{\mathrm{T}}+v_{R}^{\mathrm{T}} \tilde{C} m_{S}^{\dagger} v_{R}\right] \\
& -\frac{1}{2}\left[v_{L}^{\mathrm{T}} \tilde{C} m_{T} v_{L}+\overline{v_{L}} \tilde{C} m_{T}^{\dagger} \bar{v}_{L}^{\mathrm{T}}\right] .
\end{aligned}
$$

The mass matrices $m_{D}, m_{S}$ and $m_{T}$ are Lorentz scalars.

- $m_{D}$ is the Dirac mass.

- $m_{S}$ and $m_{T}$ violate the fermionic number, they are called Majorana mass.

The $\tilde{C}$ matrix in the Majorana mass term is used to conserve Lorentz invariance ${ }^{2}$. The matrix $C$ is necessary for the invariance of Dirac equation under a charge conjugation: $C \gamma_{\mu}^{*} C^{-1}=-\gamma_{\mu}$. In general $C$ depends on the base of the used $\gamma$ matrices. For the Majorana base, the $\gamma$ matrices are purely imaginary, so $C=1$.

$\tilde{C}$ is related to $C$ by:

$$
\tilde{C}=C \gamma^{0^{\mathrm{T}}} \Rightarrow \tilde{C} \gamma_{\mu}^{\mathrm{T}} \tilde{C}^{-1}=-\gamma_{\mu} .
$$

The reason that $\tilde{C}$ appears in Equation (17) is to connect the conjugate charge field $\psi^{c}$ to $\bar{\psi}$ and $\psi^{\dagger}$.

We have: $\psi^{c}(x)=C \psi^{\dagger}(x)$ and $\bar{\psi}=\psi^{\dagger} \gamma^{0}$, then

$$
\psi^{c}(x)=C \gamma^{0^{\mathrm{T}}} \bar{\psi}^{\mathrm{T}}(x)=\tilde{C} \bar{\psi}^{\mathrm{T}}(x) .
$$

\section{The See-Saw Mechanism}

\subsection{General Framework}

The Equation (17) describes the general mass term of neutrinos with three matrices of different masses $m_{D}, m_{S}$ and $m_{T}$ of dimension $(3 \times 3)$ since we have three flavors of neutrinos [9] [10]. This equation becomes more symmetrical if we replace the transpose field by the conjugate charge field. Using Equation (19), we obtain $\bar{\psi}^{c}=\psi^{\mathrm{T}} \tilde{C}$, then

$$
\begin{gathered}
{\overline{v_{R}}}_{\nu_{L}}=-v_{L}^{\mathrm{T}} \bar{\nu}_{R}^{\mathrm{T}}=v_{L}^{\mathrm{T}} \tilde{C} \tilde{C} \bar{\nu}_{R}^{\mathrm{T}}=\bar{\nu}_{L}^{c} v_{R}^{c}, \\
\Rightarrow \bar{v}_{R} \nu_{L}=\frac{1}{2}\left[\bar{\nu}_{R} \nu_{L}+\bar{v}_{L}^{c} v_{R}^{c}\right] .
\end{gathered}
$$

Using these equations, the Equation (17) can be written with the following compact form:

${ }^{2} \tilde{C}$ is different from the operator $\hat{C}$ which explain how a Dirac field transform under a charge conjugation. 


$$
L_{\text {masse }}^{v}=-\frac{1}{2}\left[\left(\bar{v}_{L}^{c} \bar{v}_{R}\right)\left(\begin{array}{ll}
m_{T} & m_{D}^{\mathrm{T}} \\
m_{D} & m_{S}
\end{array}\right)\left(\begin{array}{c}
v_{L} \\
v_{R}^{c}
\end{array}\right)\right]+\text { h.c. }
$$

For the 3 generations of neutrinos, the 6 eigenstates of mass $m_{i}$ are eigenvalues of the $(6 \times 6)$ matrix

$$
M=\left(\begin{array}{ll}
m_{T} & m_{D}^{\mathrm{T}} \\
m_{D} & m_{S}
\end{array}\right)
$$

$M$ is not necessarily Hermitian, so its diagonalization requires a bi-unitary transformation:

$$
U_{R}^{\dagger} M U_{L}=M_{\text {diag }},
$$

where $U_{L}$ and $U_{R}$ are $(6 \times 6)$ matrices. This diagonalization is accomplished with a change of base:

$$
\psi_{L}=\left(\begin{array}{c}
v_{L} \\
v_{R}^{c}
\end{array}\right), \quad \psi_{R}=\left(\begin{array}{c}
v_{L}^{c} \\
v_{R}
\end{array}\right),
$$

to obtain a new set of fields $\eta_{L}$ and $\eta_{R}$ defined by:

$$
\psi_{L}=U_{L} \eta_{L}, \quad \psi_{R}=U_{R} \eta_{R} .
$$

\subsection{Problem Simplification}

We can simplify our problem by working on a single neutrino family [11]. Suppose that $m_{T}=0$ and $m_{S} \gg m_{D}$, so the $(2 \times 2)$ matrix $M$ will be written as:

$$
M=\left(\begin{array}{cc}
0 & m_{D} \\
m_{D} & m_{S}
\end{array}\right) \text {. }
$$

Calculating the eigenvalues of $M$ :

$$
\operatorname{det}(M-\lambda \cdot \mathbb{I})=0 \Rightarrow \lambda^{2}-m_{S} \lambda-m_{D}^{2}=0 \Rightarrow \lambda_{ \pm}=\frac{m_{S} \pm \sqrt{m_{S}^{2}+4 m_{D}^{2}}}{2}
$$

but

$$
\sqrt{m_{S}^{2}+4 m_{D}^{2}}=m_{S}\left(1+4 \frac{m_{D}^{2}}{m_{S}^{2}}\right)^{1 / 2}=m_{S}\left(1+2 \frac{m_{D}^{2}}{m_{S}^{2}}+O(3)\right)=m_{S}+2 \frac{m_{D}^{2}}{m_{S}}
$$

therefore the eigenvalues of $M$ are $m_{S}$ and $-\frac{m_{D}^{2}}{m_{S}}$. Then the spectrum divides into a heavy neutrino of approximate mass $m_{S}$ and another light neutrino of mass $\frac{m_{D}^{2}}{m_{S}}$. This is the See-Saw mechanism.

$m_{D}$ is of the mass order of a charged lepton $\left(m_{D} \sim m_{e}\right)$ and $m_{S}$ large in front of $m_{D}$ to give small masses to the neutrinos. The $(2 \times 2)$ matrix $M$ is diagonalized by the orthogonal matrix

$$
U=\left(\begin{array}{cc}
1 & \frac{m_{D}}{m_{S}} \\
\frac{-m_{D}}{m_{S}} & 1
\end{array}\right),
$$


so the two eigenstates of mass of the two neutrinos are:

$$
\begin{gathered}
\eta_{L}=\left(\begin{array}{l}
\eta_{1} \\
\eta_{2}
\end{array}\right)_{L}=\left(\begin{array}{cc}
1 & \frac{-m_{D}}{m_{S}} \\
\frac{m_{D}}{m_{S}} & 1
\end{array}\right)\left(\begin{array}{l}
v_{L} \\
v_{R}^{c}
\end{array}\right)=\left(\begin{array}{c}
v_{L}-\frac{m_{D}}{m_{S}} v_{R}^{c} \\
v_{R}^{c}+\frac{m_{D}}{m_{S}} v_{L}
\end{array}\right), \\
\eta_{R}=\left(\begin{array}{l}
\eta_{1} \\
\eta_{2}
\end{array}\right)_{R}=\left(\begin{array}{cc}
1 & \frac{-m_{D}}{m_{S}} \\
\frac{m_{D}}{m_{S}} & 1
\end{array}\right)\left(\begin{array}{l}
v_{L}^{c} \\
v_{R}
\end{array}\right)=\left(\begin{array}{c}
v_{L}^{c}-\frac{m_{D}}{m_{S}} v_{R} \\
v_{R}+\frac{m_{D}}{m_{S}} v_{L}^{c}
\end{array}\right) .
\end{gathered}
$$

We note that the two eigenstates of mass $\eta_{1}$ and $\eta_{2}$ are Majorana states:

$$
\begin{aligned}
& \eta_{1}=\eta_{1 L}+\eta_{1 R}=\left(v_{L}+v_{L}^{c}\right)-\frac{m_{D}}{m_{S}}\left(v_{R}+v_{R}^{c}\right)=\eta_{1}^{c}, \\
& \eta_{2}=\eta_{2 L}+\eta_{2 R}=\left(v_{R}+v_{R}^{c}\right)+\frac{m_{D}}{m_{S}}\left(v_{L}+v_{L}^{c}\right)=\eta_{2}^{c} .
\end{aligned}
$$

The state $v_{L}$ which undergoes essentially the weak interactions is $\eta_{1 L}$; it is the state associated to the eigenstate of the light neutrino $\left(m_{1} \simeq \frac{m_{D}^{2}}{m_{S}}\right)$ :

$$
v_{L}=\eta_{1 L}+\frac{m_{D}}{m_{S}} \eta_{2 L}
$$

The right neutrino $v_{R}$ is the eigenstate of the heavy neutrino $\eta_{2 R}$, $\left(m_{2}=m_{S}\right)$ :

$$
v_{R}=\eta_{2 R}-\frac{m_{D}}{m_{S}} \eta_{1 R}
$$

This example can be generalized by neglecting the matrix $m_{T}$ (its eigenvalues are negligible), then the neutrino mass matrix takes the form:

$$
M=\left(\begin{array}{cc}
0 & m_{D}^{\mathrm{T}} \\
m_{D} & m_{S}
\end{array}\right)
$$

If $m_{S} \gg m_{D}$, then we also obtain a spectrum which separates into a light neutrino and a heavy one. The light neutrino has a $M(3 \times 3)$ light mass matrix:

$$
\left(M_{v}\right)_{\text {light }}=m_{D}^{\mathrm{T}} m_{S}^{-1} m_{D},
$$

and the heavy neutrino:

$$
\left(M_{v}\right)_{\text {heavy }}=m_{S} \text {. }
$$

The eigenstates obtained are of Majorana type. The eigenvalues of mass for the left neutrinos are given by:

$$
m_{k}=\frac{\left(m_{k}^{f}\right)^{2}}{m_{S}} \quad(k=1,2,3),
$$

where $m_{k}^{f}$ is the mass of a quark or a charged lepton of generation $k$. The left neutrino acquires a mass inversely proportional to the mass of the right neutrino. 
So the See-Saw mechanism explains, not only, the small mass of the left neutrinos, but it also introduces massive Majorana-type neutrinos.

\section{Conclusion}

In the present work we derived the Dirac equation for neutrinos, which represents a relativistic quantum mechanics equation that describes half-integer spin particles. Then we introduced the concept of Majorana neutrinos using the charge conjugate operator. After that, we calculated in details the general mass terms for both types of neutrinos. Finally, we presented the general idea of the See-Saw mechanism and we calculated the mass eigenstates of the Dirac and Majorana neutrinos. We also explained how a spectrum separates into a light and heavy neutrinos.

\section{Conflicts of Interest}

The authors declare no conflicts of interest regarding the publication of this paper.

\section{References}

[1] Rajasekaran, G. (2016) The Story of the Neutrino. arXiv:1606.08715 [physics.pop-ph]

[2] Kayser, B. (2008) Neutrino Mass, Mixing, and Flavor Change. arXiv:0804.1497 [hep-ph]

[3] de Gouvea, A. (2004) TASI Lectures on Neutrino Physics.. hep-ph/0411274

[4] Peccei, R.D. (1999) Neutrino Physics. AIP Conference Proceedings, 490, 80. [hep-ph/9906509] https://doi.org/10.1063/1.1301383

[5] Ryder, L.H. (1996) Quantum Field Theory.

[6] Fritzsch, H. (2020) Oscillating Neutrinos and Majorana Neutrino Masses. Universe, 6, 29. [arXiv:2002.00267 [hep-ph]] https://doi.org/10.3390/universe6020029

[7] Battye, R.A. and Moss, A. (2014) Evidence for Massive Neutrinos from Cosmic Microwave Background and Lensing Observations. Physical Review Letters, 112, 051303. [arXiv:1308.5870 [astro-ph.CO]] https://doi.org/10.1103/PhysRevLett.112.051303

[8] Raidal, M., et al. (2008) Flavor Physics of Leptons and Dipole Moments. The European Physical Journal C, 57, 13 [arXiv:0801.1826 [hep-ph]] https://doi.org/10.1140/epjc/s10052-008-0715-2

[9] Mohapatra, R.N., et al. (2007) Theory of Neutrinos: A White Paper. Reports on Progress in Physics, 70, 1757. [hep-ph/0510213] https://doi.org/10.1088/0034-4885/70/11/R02

[10] Akhmedov, E.K., Branco, G.C. and Rebelo, M.N. (2000) Physics Letters B, 478, 215. [hep-ph/9911364] https://doi.org/10.1016/S0370-2693(00)00282-3

[11] Mohapatra, R.N. (2004) Seesaw Mechanism and Its Implications. hep-ph/0412379 